\title{
Capture of dengue viruses using antibody-integrated graphite-encapsulated magnetic beads produced using gas plasma technology
}

\author{
AKIKAZU SAKUDO ${ }^{1}$, ANCHU VISWAN $^{2}$, HAN CHOU $^{3}$, TADAHIRO SASAKI $^{4}$, \\ KAZUYOSHI IKUTA ${ }^{4,5}$ and MASAAKI NAGATSU ${ }^{2,3}$
}

\begin{abstract}
${ }^{1}$ Laboratory of Biometabolic Chemistry, School of Health Sciences, Faculty of Medicine, University of the Ryukyus, Nishihara, Okinawa 903-0215; ${ }^{2}$ Department of Nanovision Technology, Graduate School of Science and Technology;

${ }^{3}$ Department of Electrical and Electronic Engineering, Graduate School of Engineering, Shizuoka University, Naka-Ku, Hamamatsu 432-8561; ${ }^{4}$ Department of Virology, Research Institute for Microbial Diseases, Osaka University, Osaka 565-0871; ${ }^{5}$ Research and Development Division, Kanonji Institute, The Research Foundation for Microbial Diseases of Osaka University, Kanonji, Kagawa 768-0061, Japan
\end{abstract}

Received July 10, 2015; Accepted May 9, 2016

DOI: $10.3892 / \mathrm{mmr} .2016 .5330$

\begin{abstract}
Despite significant advances in medicine, global health is threatened by emerging infectious diseases caused by a number of viruses. Dengue virus (DENV) is a mosquito-borne virus, which can be transmitted to humans via mosquito vectors. Previously, the Ministry of Health, Labour and Welfare in Japan reported the country's first domestically acquired case of dengue fever for almost 70 years. To address this issue, it is important to develop novel technologies for the sensitive detection of DENV. The present study reported on the development of plasma-functionalized, graphite-encapsulated magnetic nanoparticles (GrMNPs) conjugated with anti-DENV antibody for DENV capture. Radiofrequency wave-excited inductively-coupled Ar and ammonia gas plasmas were used to introduce amino groups onto the surface of the GrMNPs. The GrMNPs were then conjugated with an antibody against DENV, and the antibody-integrated magnetic beads were assessed for their ability to capture DENV. Beads incubated in a cell culture medium of DENV-infected mosquito cells were separated from the supernatant by applying a magnetic field and were then washed. The adsorption of DENV serotypes 1-4 onto the beads was confirmed using reverse transcription-polymerase chain reaction, which detected the presence of DENV genomic RNA on the GrMNPs. The methodology described in the present study, which employed the plasma-functionalization of
\end{abstract}

Correspondence to: Dr Akikazu Sakudo, Laboratory of Biometabolic Chemistry, School of Health Sciences, Faculty of Medicine, University of the Ryukyus, 207 Uehara, Nishihara, Okinawa 903-0215, Japan

E-mail: sakudo@med.u-ryukyu.ac.jp

Key words: graphite, antibody, gas plasma, virus concentration, dengue, virus capture
GrMNPs to enable antibody-integration, represents a significant improvement in the detection of DENV.

\section{Introduction}

Dengue virus (DENV), a plus-strand RNA virus with an enveloped icosahedral nucleocapsid, can be transmitted through mosquito vectors, and is the causative agent of dengue fever, dengue hemorrhagic fever and dengue shock syndrome (1). Dengue hemorrhagic fever and dengue shock syndrome are potentially life threatening, and the risk of developing these diseases is correlated with infection by one of the four DENV serotypes (DENV1-4) and the carrying of antibodies to another DENV serotype from a previous infection (2). Currently, no specific treatment or vaccine for DENV is available (3).

There has been a significant increase in the number of reports of DENV-associated infections and DENV-associated mortality (1). Notably, over the last 50 years, the incidence of DENV-associated infections has increased by 30 -fold, and the World Health Organization estimate that there are currently $50,000,000$ cases per annum worldwide (3). Infections due to DENV are now of serious concern worldwide, particularly in subtropical areas, including Southeast Asia (4). DENV was first isolated by Kimura and Hotta from blood samples obtained during the 1943 dengue epidemic, which was predominantly confined to the Japanese port cities of Nagasaki, Kobe and Osaka (5). On the 28th August 2014, the Ministry of Health, Labour and Welfare in Japan reported the country's first domestically acquired case of dengue fever for almost 70 years (6). Therefore, the implementation of procedures to monitor for any further potential outbreak of dengue fever in the country are required.

Consequently, the development of effective methods for the surveillance of DENV are urgently required. One approach to achieve sensitive detection of DENV is to establish a method to concentrate the viral particles. Several approaches 
to concentrate virus have been suggested, including ultracentrifugation and polyethylene glycol (PEG)-mediated precipitation. Although these methods are applicable to a number of viruses, they have significant practical limitations. Specifically, ultracentrifugation is time-consuming and can increase the false-positive rate when combined with polymerase chain reaction (PCR) analysis (7). Although PEG-mediated precipitation is simple and easy to perform, PEG interferes with the subsequent PCR procedure $(8,9)$. An alternative to these conventional methods is the use of magnetic beads coated with molecules, which efficiently bind to the virus, which allows the capture and concentration of the viral particles by applying a magnetic field. A potential approach to the capture of a target virus is to use magnetic beads coated with an antibody specific to the particular virus of interest.

Magnetic nanoparticles (MNPs), including iron, nickel or cobalt, have been widely investigated for biomedical and environmental applications due to their high specific surface area and the ease of magnetic collection of target materials adsorbed by the MNPs $(10,11)$. However, a significant problem with these beads is their inherent chemical instability, which can limit their application in the field of biological and environmental science (12). To overcome this limitation, the MNPs are typically encapsulated with a protective shell of graphite, silica or polymer (12).

Graphite-encapsulated MNPs (GrMNPs) are usually hydrophobic, which is a limitation for several biomedical applications. However, appropriate surface modification can improve the properties of the GrMNPs, allowing them to efficiently recognize and bind to molecular targets, including antibodies, antigens and receptors (13). Amino group functionalization, which is a desirable functionality for graphite, improves the reactivity and hydrophilic nature of the GrMNPs (12). A promising method for the amino functionalization of GrMNPs is to use inductively coupled radiofrequency ( $R F$ ) plasma, which is environmentally friendly and requires a short duration for the reaction to reach completion. Using this approach, it is possible to introduce amino groups effectively (14). Furthermore, the degree of surface derivatization with amino groups of GrMNPs can be optimized by altering the plasma discharge conditions (15). With these amino-modified GrMNPs, the efficient surface immobilization of sugar chains has been demonstrated, including dextran, as well as antibodies against several pathogens, including anti-influenza virus and anti-Salmonella antibodies $(12,16,17)$.

On the basis of this background, the present study was performed to expand on previous results examining the influenza virus to investigate DENV via the immobilization of anti-DENV antibody onto the functionalized surface of GrMNPs. The modified GrMNPs were then assessed for their ability to capture DENVs, and the concentrated virus was then detected in combination with a PCR-based amplification procedure.

\section{Materials and methods}

Plasma-functionalized GrMNPs and production of antibody-integrated magnetic beads. The graphite-encapsulated iron compound nanoparticles were prepared using an arc discharge method by applying a 150-200 A direct current at $\sim 20 \mathrm{~V}$ between an anode and cathode, as described previously (15). A graphite electrode, molded using graphibond-551R with $\mathrm{Fe}_{2} \mathrm{O}_{3}$ powder, was used as the anode. On the opposite side, a graphite $\operatorname{rod}(50 \mathrm{~mm} \bullet \varnothing 10 \mathrm{~mm}$; $99.9 \%)$ was used as the cathode. The resulting graphite-encapsulated iron compound nanoparticles were then exposed to plasma, which was produced using an RF power supply $(18,19)$ in an atmosphere containing ammonia at $13.56 \mathrm{MHz}$ and $80 \mathrm{~W}$ via a matching network $(18,19)$. Initial pretreatment was performed for $10 \mathrm{~min}$ using Ar plasma, followed by 2 min of ammonia plasma post-treatment for amino group introduction. During the experiments, the gas pressure was maintained at $50 \mathrm{~Pa}$. The amino groups on the surface of the magnetic beads were then further labelled with $0.3 \mu \mathrm{M}$ of the coupling agent, $\mathrm{N}$-succinimidyl 3-(2-pyridyldithio) propionate (SPDP; Dojindo Laboratories, Kumamoto, Japan) at pH 7-8. A human monoclonal antibody (clone no. D23-1G7C2) recognizing the first domain II fusion region of the DENV envelope glycoprotein (E) (20) was reduced using dithiothreitol (DTT), resulting in breakage of the S-S bonds and generation of S-H groups. The D23-1G7C2 antibody was produced from hybridomas using peripheral blood mononuclear cells from patients in the acute phase of dengue fever 5 days following the onset of illness, and exhibits neutralizing activity against DENV1-4 (20). The S-H groups on the antibody were then reacted with the SPDP- $\mathrm{NH}_{2}$-magnetic beads, resulting in covalent crosslinking of the antibody onto the surface of the beads. The resulting magnetic beads were termed antibody-integrated magnetic beads (Fig. 1).

Cell culture and virus. A C6/36 cell culture (American Type Culture Collection, Manassas, VA, USA), derived from Aedes albopictus, was maintained in Leibovitz L15 medium (Thermo Fisher Scientific, Inc., Waltham, MA, USA) containing $0.3 \%$ tryptose phosphate broth (TPB) and $10 \%$ fetal calf serum (FCS; Wako Pure Chemical Industries, Ltd., Osaka, Japan). The laboratory DENV strains (21), DENV1 (Mochizuki strain), DENV2 (16681 strain), DENV3 (80-2 strain) and DENV4 (H241 strain), were used to infect the $\mathrm{C} 6 / 36$ cell cultures. The $\mathrm{C} 6 / 36$ cells were cultured to $\sim 80 \%$ confluence, then infected with the DENVs at a multiplicity of infection of 0.1 in Leibovitz L15 medium containing $0.3 \% \mathrm{TPB}$ and $2 \% \mathrm{FCS}$, and were incubated for 3 days at $28^{\circ} \mathrm{C}$. The medium was then collected and used for viral capture experiments.

DENV capture. The capture of DENV1-4 was performed as follows. Briefly, $10 \mu \mathrm{l}$ of the magnetic beads were washed twice with phosphate-buffered saline (PBS). A $10 \mu \mathrm{l}$ sample of medium from uninfected (Mock) or DENV-infected cell cultures were added to the washed beads with $1 \mathrm{ml}$ PBS, and the tube was incubated for $15 \mathrm{~min}$ at room temperature. The tubes containing the mixtures were then set in a magnetic field for 5 min using an Adem-Mag SV magnetic device (Ademtech, Pessac, France). Following magnetic separation, the supernatant was removed, and the beads were washed three times with PBS and resuspended in $10 \mu \mathrm{l}$ PBS. This procedure produced two fractions: Bead fraction (BD) and supernatant fraction 

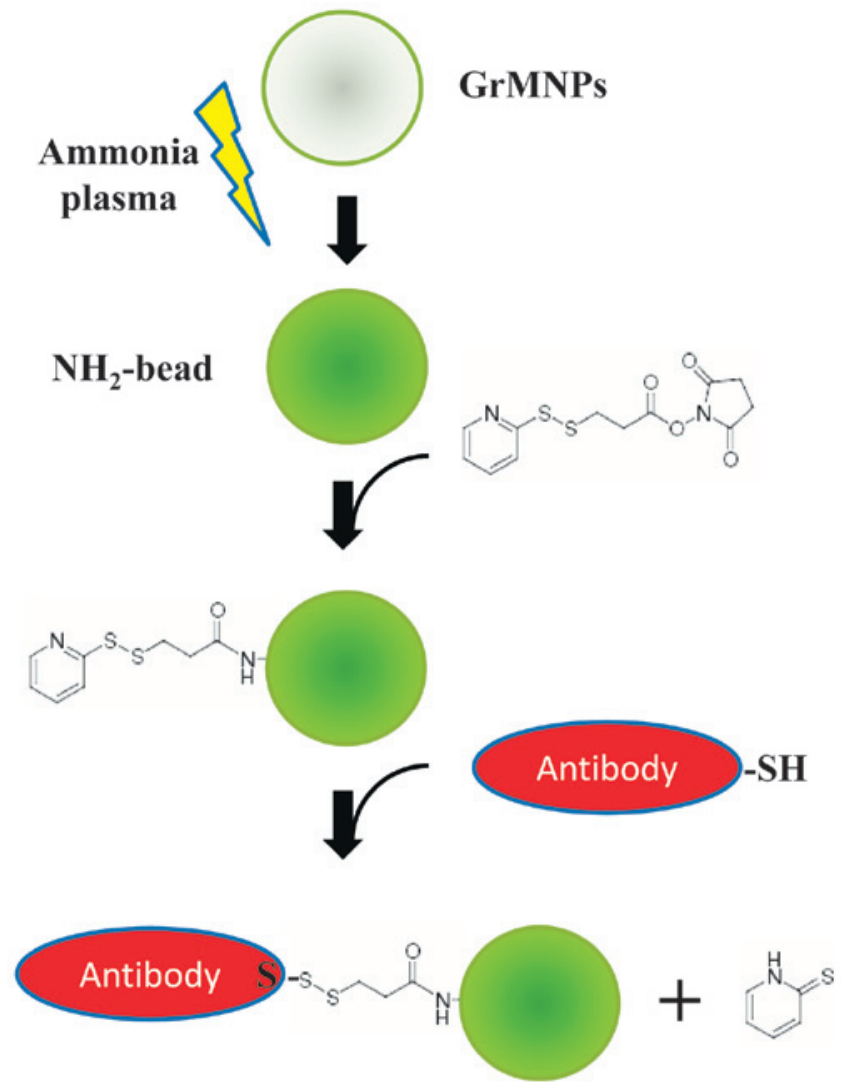

Figure 1. Schematic representation of the ammonia plasma-treated GrMNPs and their binding to anti-DENV antibody, resulting in production of antibody-integrated magnetic beads. The surfaces of the GrMNPs were reacted with ammonia plasma, produced using a radiofrequency power supply, resulting in the introduction of amino groups. SPDP was reacted with the amino group-functionalized magnetic beads $\left(\mathrm{NH}_{2}\right.$-beads) at $\mathrm{pH}$ 7-8. Anti-DENV antibody (D23-1G7C2) was reduced using dithiothreitol, resulting in the breakage of S-S-bonds and the generation of S-H groups. The S-H group of the antibody was then further reacted with the SPDP- $\mathrm{NH}_{2}$-magnetic beads. The resultant magnetic beads were termed antibody-integrated magnetic beads. GrMNPs, graphite-encapsulated magnetic nanoparticles; DENV, dengue virus; SPDP, $N$-succinimidyl 3-(2-pyridyldithio) propionate.

(SP). A $10 \mu 1$ sample of each fraction was used for reverse transcription (RT)-PCR analysis.

RT-PCR analysis. The DENV genomic RNA from each fraction was extracted using a QIAamp Viral RNA Mini kit (Qiagen, Hilden, Germany), according to the manufacturer's protocol. The RT reactions were performed using a PrimeScriptII First UStrand cDNA synthesis kit (Takara Bio, Inc., Otsu, Japan) with random primers. Following incubation at $65^{\circ} \mathrm{C}$ for $5 \mathrm{~min}$, the viral RNA was reverse transcribed at $42^{\circ} \mathrm{C}$ for $60 \mathrm{~min}$. The resultant cDNA $(2 \mu \mathrm{l})$ was amplified by PCR, in a reaction mixture $(20 \mu \mathrm{l})$ containing primers $(0.2 \mu \mathrm{l}$ each, $100 \mathrm{pmol} / \mu \mathrm{l})$, $\mathrm{MgCl}_{2}(1.6 \mu \mathrm{l}, 25 \mathrm{mM})$, dNTP mixture (1.6 $\mu \mathrm{l}, 2.5 \mathrm{mM}$ each), Ex Taq $(0.1 \mu \mathrm{l}, 5 \mathrm{U} / \mu \mathrm{l}$; Takara Bio, Inc.) and 10X Ex Taq buffer $(2 \mu \mathrm{l})$ under conditions of 35 cycles at $94^{\circ} \mathrm{C}$ for $30 \mathrm{sec}$, $55^{\circ} \mathrm{C}$ for $30 \mathrm{sec}$ and $72^{\circ} \mathrm{C}$ for $1 \mathrm{~min}$. The primers used were as follows: D1, forward 5'-tcaatatgctgaaacgcgggagaaaccg-3' and D2, reverse 5'-ttgcaccaacagtcaatgtcttcaggttc-3' (22). The amplified DNA fragments were purified and analyzed by DNA sequencing on an ABI PRISM3100 genetic analyzer (Applied
Biosystems; Thermo Fisher Scientific, Inc.) to verify the identity of the amplified product. Then, the product sequences were compared with the sequences in the Genbank database (ncbi.nlm.nih.gov/genbank/).

Amino group detection using fluorescamine. Fluorescamine reacts with amino groups and forms blue-green fluorescent derivatives. In the present study, fluorescence intensity increased in a dose-dependent manner with bovine serum albumin (BSA) concentrations following incubation with fluorescamine (Wako Pure Chemical Industries, Ltd.) at an excitation of $400 \mathrm{~nm}$ and emission of $490 \mathrm{~nm}$. The amino groups on the GrMNPs treated with ammonia plasma were measured using a fluorometric method with fluorescamine, in accordance with a previous study with modification (23). Briefly, $5 \mu \mathrm{l}$ of the plasma-treated nanoparticles $(2 \mathrm{mg} / \mathrm{ml})$ were solubilized with $100 \mu 150 \mathrm{mM}$ borate buffer, following which $4 \mu 10.075 \%$ fluorescamine in acetonitrile was added to each sample. The fluorescence, at an emission of $490 \mathrm{~nm}$ and excitation of $400 \mathrm{~nm}$, was measured using a SpectraMax M2e HK fluorometer (Molecular Devices, Sunnyvale, CA, USA).

Amino group detection using 2,4,6-trinitrobenzenesulfonic acid (TNBS). TNBS reacts with amino groups and shows an increase in absorbance at $\sim 350 \mathrm{~nm}$ (24). When $10 \mathrm{mg} / \mathrm{ml}$ BSA was reacted with TNBS (Wako Pure Chemical Industries, Ltd.), the maximal peak of the spectra was $345 \mathrm{~nm}$. In addition, a dose-dependent increase of the corresponding peak in BSA was observed following the TNBS reaction at $345 \mathrm{~nm}$. The amino groups on the GrMNPs treated with ammonia plasma were measured using a colorimetric method using TNBS in accordance with a previous study with modification (24). Briefly, $20 \mu \mathrm{l}$ of the plasma-treated GrMNPs $(2 \mathrm{mg} / \mathrm{ml})$ in $50 \mathrm{mM}$ borate buffer was incubated for $16 \mathrm{~h}$ at $4^{\circ} \mathrm{C}$ following the addition of $0.5 \%$ TNBS. Subsequently, $250 \mu \mathrm{l}$ of $5 \%$ sodium dodecyl sulfate and $2 \mathrm{~N} \mathrm{HCl}$ were added, and the absorbance at $345 \mathrm{~nm}$ was measured using a spectrophotometer (UVmini-1240; Shimadzu Corporation, Kyoto, Japan).

RT-PCR for DENV. Viral genomic RNA was extracted from the samples prior to and following magnetic separation using a QIAamp Viral RNA mini kit (Qiagen). The RNA was reverse transcribed using a PrimeScriptII first strand cDNA synthesis kit (Takara Bio, Inc.) using the following temperature regime: $65^{\circ} \mathrm{C}$ for $5 \mathrm{~min}, 4^{\circ} \mathrm{C}$ for $5 \mathrm{~min}$ and $42^{\circ} \mathrm{C}$ for $60 \mathrm{~min}$. The resulting cDNAs were subjected to PCR using SYBR Premix Ex TaqII (Tli RNase H Plus; Takara Bio, Inc.). The primers for DENV were as follows: forward $5^{\prime}-\operatorname{ct}(\mathrm{a}, \mathrm{t}) \mathrm{tca}$ atatgctgaaac gcg-3' and reverse 5'-tctatcca(g,a)aat (t,c)cctgctgtt-3' (22). The temperature cycling conditions used for PCR were as follows: $95^{\circ} \mathrm{C}$ for $30 \mathrm{sec}$, followed by 40 cycles of $95^{\circ} \mathrm{C}$ for $5 \mathrm{sec}$ and $60^{\circ} \mathrm{C}$ for $1 \mathrm{~min}$.

Statistical analysis. Statistical analyses were performed using Prism 4 software (GraphPad Software, Inc., San Diego, CA, USA). The TNBS and fluorescamine data were subjected to a non-repeated measures analysis of variance followed by the Bonferroni correction test. The PCR data were subjected to Student's $t$-test (unpaired). $\mathrm{P}<0.05$ is considered to indicate a statistically significantly difference. 
Table I. Confirmation of amino groups on the surface of ammonia plasma-treated GrMNPs by reaction with TNBS.

Treatment

Absorbance at $345 \mathrm{~nm}$

Buffer + TNBS

Ammonia-plasma-treated GrMNPs + TNBS

$0.2973 \pm 0.002$

Supernatant of ammonia-plasma-treated GrMNPs + TNBS (post-centrifugation)

$0.8153 \pm 0.004^{\mathrm{a}}$

BSA $(0 \mathrm{mg} / \mathrm{ml})$

$0.6833 \pm 0.003^{\mathrm{a}}$

$\operatorname{BSA}(0.1 \mathrm{mg} / \mathrm{ml})$

0.295

$\operatorname{BSA}(1 \mathrm{mg} / \mathrm{ml})$

0.327

$\operatorname{BSA}(10 \mathrm{mg} / \mathrm{ml})$

0.499

1.745

TNBS was used to detect amino groups on ammonia plasma-treated GrMNPs. TNBS was incubated with either the ammonia plasma-treated GrMNPs in borate buffer prior to centrifugation, the supernatant following centrifugation, or with borate buffer alone. The absorbance at $345 \mathrm{~nm}$ was then measured. As a positive control, samples of $0-10 \mathrm{mg} / \mathrm{ml} \mathrm{BSA}$ were incubated with TNBS for 5 min and their absorbance measured. The absorbance of the ammonia plasma-treated GrMNPs + TNBS prior to and following centrifugation was significantly higher than that of the buffer + TNBS group, indicating TNBS had reacted with the amino groups on the plasma-treated GrMNPs. BSA measurements were obtained as positive controls $(\mathrm{n}=1)$. ${ }^{\mathrm{a}} \mathrm{P}<0.05$, compared with buffer + TNBS. Values are presented as the mean \pm standard error. GrMNPs, graphite encapsulated magnetic beads; TNBS, 2,4,6-trinitrobenzenesulfonic acid; BSA, bovine serum albumin.

Table II. Confirmation of amino groups on the surface of ammonia plasma-treated GrMNPs by reaction with fluorescamine.

Treatment

Fluorescence (excitation $400 \mathrm{~nm}$; emission $490 \mathrm{~nm}$ )

Ammonia-plasma-treated GrMNPs

$112.49 \pm 7.71^{\mathrm{a}}$

Antibody-integrated magnetic beads

$101.00 \pm 7.29^{\mathrm{a}}$

BSA $(0 \mathrm{mg} / \mathrm{ml})$

$84.23 \pm 2.55$

$\operatorname{BSA}(0.1 \mathrm{mg} / \mathrm{ml})$

$178.28 \pm 15.88^{\mathrm{a}}$

$\mathrm{BSA}(1 \mathrm{mg} / \mathrm{ml})$

$862.58 \pm 5.70^{\mathrm{a}}$

Fluorescamine was incubated with either ammonia plasma-treated GrMNPs in borate buffer or borate buffer alone, and fluorescence was measured. As a positive control, samples of $0-1 \mathrm{mg} / \mathrm{ml}$ BSA were incubated with fluorescamine for 5 min and their fluorescence signals were measured. The results confirmed the amino groups reacted with fluorescamine. The fluorescence signals of the ammonia plasma-treated GrMNPs and anti-dengue virus antibody-integrated magnetic beads were significantly higher, compared with the borate buffer alone. These observations indicated that fluorescamine had reacted with the amino groups present on the surface of the plasma-treated GrMNPs. ${ }^{a} \mathrm{P}<0.05$, compared with $0 \mathrm{mg} / \mathrm{ml}$ BSA. Values are presented as the mean \pm standard error. GrMNPs, graphite encapsulated magnetic beads; BSA, bovine serum albumin.



Figure 2. Aggregation and suspension of ammonia plasma-treated GrMNPs following incubation with SPDP. A $0.2 \mathrm{mg} / \mathrm{ml}$ suspension of amino group-functionalized magnetic beads ( $\mathrm{NH}_{2}$-beads) was incubated with 0.03 , $0.3,3$ or $30 \mu \mathrm{M}$ SPDP for $30 \mathrm{~min}$ at room temperature. Aggregation of the beads was observed following incubation with $30 \mu \mathrm{M}$ SPDP, whereas the beads were in suspension in the $0.03,0.3$ and $3 \mu \mathrm{M}$ SPDP solutions. These observations indicated the appropriate concentration of SPDP for the suspension of beads and suggested they exhibited hydrophilic properties due the presence of amino groups at their surface. GrMNPs, graphite-encapsulated magnetic nanoparticles; SPDP, $N$-succinimidyl 3-(2-pyridyldithio) propionate.

\section{Results}

In order to analyze the hydrophilicity of the ammonia plasma-treated GrMNPs, the resultant $\mathrm{NH}_{2}$-beads were incubated with various concentrations of SPDP (Fig. 2). The results showed that the beads aggregated following incubation with $30 \mu \mathrm{M}$ SPDP, whereas the beads incubated with 0.03 , 0.3 and $3 \mu \mathrm{M}$ SPDP remained in suspension. These observations suggested that the ammonia plasma-treated GrMNPs possessed hydrophilic properties, possibly due to the introduction of amino groups to the bead surface.

To further confirm the presence of amino groups on the surface of the beads, the ammonia plasma-treated GrMNPs were incubated with TNBS or fluorescamine, which react with an amine functionality (Tables I and II). BSA, which has a surface exposed amino groups, was also reacted with TNBS or fluorescamine. As expected, an increase in the absorbance of TNBS at $345 \mathrm{~nm}$ and in the fluorescent intensity of fluorescamine (excitation $400 \mathrm{~nm}$; emission $490 \mathrm{~nm}$ ) was observed 


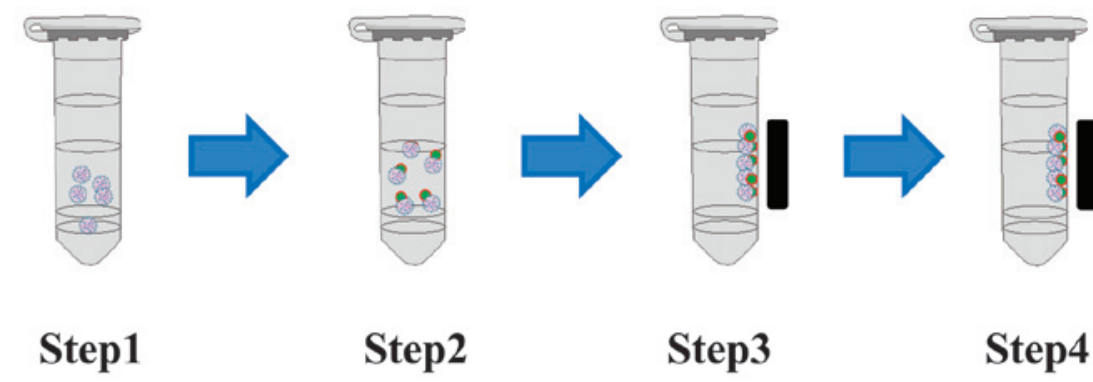

\section{Antibody-integrated magnetic beads}

\section{DENV}

\section{Magnet}

Figure 3. Schematic representation showing the capture of DENVs using antibody-integrated magnetic beads. Antibody-integrated magnetic beads (10 $\mu 1)$ were washed twice with PBS. The cell culture medium (10 $\mu 1)$ of the C6/36 mosquito cells infected with DENV1-4 was diluted with 1 ml PBS and used as DENV suspension (Step 1). The suspension was then incubated with magnetic beads for 15 min at room temperature (Step 2). Tubes containing the magnetic beads were placed in a magnetic field (Step 3). The beads were then subjected to magnetic separation, and the supernatant was removed (Step 4). The beads were washed three times with PBS and resuspended in $10 \mu 1$ PBS for further analysis. Steps 1-4 produced a bead fraction (10 $\mu 1$ antibody-integrated magnetic beads following incubation with PBS-diluted DENV-containing cell culture medium), and s supernatant fraction (10 $\mu 1$ supernatant; following incubation and washing). GrMNPs, graphite-encapsulated magnetic nanoparticles; DENV, dengue virus; PBS, phosphate-buffered saline.

\section{Mock DENV1 DENV2 DENV3 DENV4 \\ M SP BD SP BD SP BD SP BD SP BD}

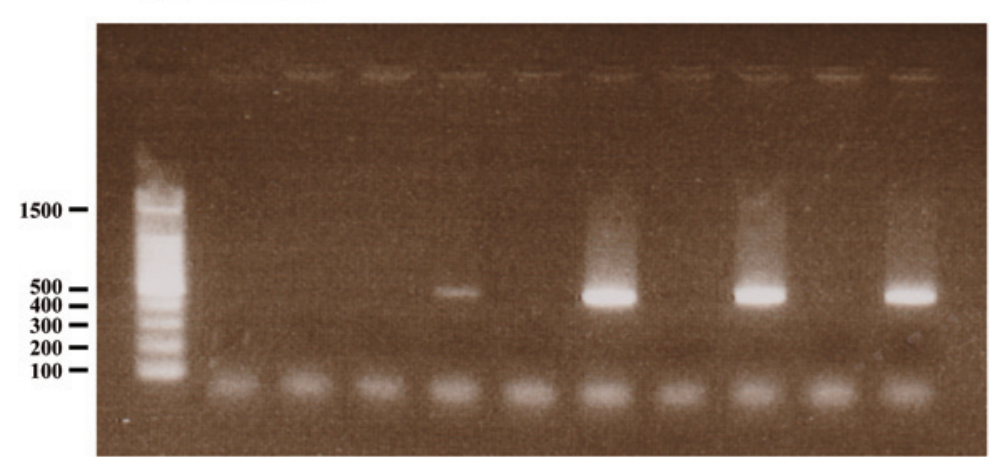

Figure 4. Detection of genomic RNA in DENVs adsorbed onto antibody-integrated magnetic beads. Culture media containing uninfected (Mock) C6/36 cells or C6/36 cells infected with DENV1-4 were mixed with antibody-integrated magnetic beads and separated into BD and SP fractions. Viral RNA was extracted from each fraction using a QIAamp Viral RNA Mini kit and analyzed using RT-PCR analysis. The PCR primer pairs specifically annealed to a common 511 bp fragment among the DENV1-4 serotypes 1-4. Each PCR product was analyzed using agarose gel electrophoresis, and the amplified products were then sequenced to confirm they corresponded to the gene of the DENVs. A 100 bp ladder marker (M) is shown. Numbers indicated on the left are molecular size (bp). DENV, dengue virus; BD, bead fraction; SP, supernatant fraction; RT-PCR, reverse transcription-polymerase chain reaction.

following incubation of BSA with the respective reagents. Subsequently, the ammonia plasma-treated GrMNPS were exposed to either TNBS or fluorescamine. The ammonia plasma-treated GrMNPs exhibited increased absorbance at $345 \mathrm{~nm}$ and increased fluorescent intensity (excitation 400 $\mathrm{nm}$; emission $490 \mathrm{~nm}$ ) following incubation with TNBS and fluorescamine, respectively. Following incubation with TNBS, the samples were centrifuged for $5 \mathrm{~min}$ at 20,000 x $\mathrm{g}$ and room temperature, and the resultant supernatant also showed increased absorbance at $345 \mathrm{~nm}$, indicating that the increased absorbance was not due to turbidity. Taken together, these results suggested that the ammonia plasma-treated GrMNPs possessed surface-exposed amino groups.

Subsequently, the present study examined the functionality of the resultant antibody-integrated magnetic beads by incubating them with a suspension of DENV prior to separation of the beads by applying a magnetic field to confirm capture of the viral particles (Fig. 3). Specifically, media from C6/36 mosquito cells infected with DENV1-4 were diluted with PBS and mixed with the magnetic beads. The mixture was then magnetically separated into the BD and supernatant SP fractions (Fig. 4). Medium from mock-infected cells was used to prepare the 
control fractions. Finally, the fractions were analyzed using RT-PCR to determine the extent of DENV capture by the beads.

Using primers specific for a 511 bp section of DENV RNA, RT-PCR was used to amplify a product of the expected size in the BD fraction, but not in the SP fraction in DENV1-4 (Fig. 4). Two independent DNA sequence analyses were performed to confirm the identity of the $511 \mathrm{bp}$ band. In each case, within the sequenced region, the following results were obtained using the Genbank database: DENV1 (Mochizuki strain) exhibited 98 and 99\% sequence identity to Genbank accession no. AB074760; DENV2 (16681 strain) exhibited 98 and 99\% sequence identity to Genbank accession no. U87411; DENV3 (80-2 strain) exhibited 95 and $95 \%$ sequence identity to Genbank accession no. AF317645; and DENV4 (H241 strain) exhibited 99 and 99\% sequence identity to Genbank accession no. AY947539.

Subsequently, viral RNA was extracted, transcribed and subjected to RT-PCR using specific forward and reverse primers for DENV in order to investigate the quantity of DENV genomic RNA prior to and following magnetic separation (Fig. 5). The percentage of genomic RNA in DENV1 recovered following magnetic separation by the antibody-integrated magnetic beads was $310.7 \pm 46.0 \%$, compared with the control. Of note, this sample was the same as the BD fraction. By contrast, the percentage in the sample prior to incubation with the beads was $97.3 \pm 11.2 \%$, compared with the control. These findings suggested that the antibody-integrated magnetic beads concentrated the DENV1 by 3.2-fold.

The above results confirmed that the BD fraction contained the corresponding DENV genomic RNAs, confirming that the beads had successfully captured and concentrated the DENV particles.

\section{Discussion}

Our previous studies showed that influenza virus, an enveloped RNA virus, and Salmonella enterica, a pathogenic bacterium associated with food poisoning can be efficiently captured using amino modified GrMNPs, generated by an ammonia gas plasma mediated strategy, coated with the corresponding antibody $(16,17)$. Therefore, the generic strategy of using ammonia gas plasma to introduce amino groups into GrMNP, and then covering the beads with antibodies directed against a specific bacteria, virus or other pathogen, facilitates their efficient capture from liquid samples. The targeted pathogen is then pre-concentrated using immunomagnetic separation, enabling their identification using a suitable detection procedure, for example PCR. This method offers potential as an effective monitoring tool for emerging viruses and other pathogens by facilitating their rapid and sensitive detection. Therefore, the method described in the present study contributed to controlling current and future global infectious threats in the areas of food, medical and environmental science.

The efficiency of introducing amino groups onto the bead surface using the ammonia gas plasma technique may be increased by carefully adjusting the treatment conditions. Although the mechanism underlying the attachment of amino groups via plasma treatment remains to be fully elucidated, its clarification may further enhance the efficient introduction

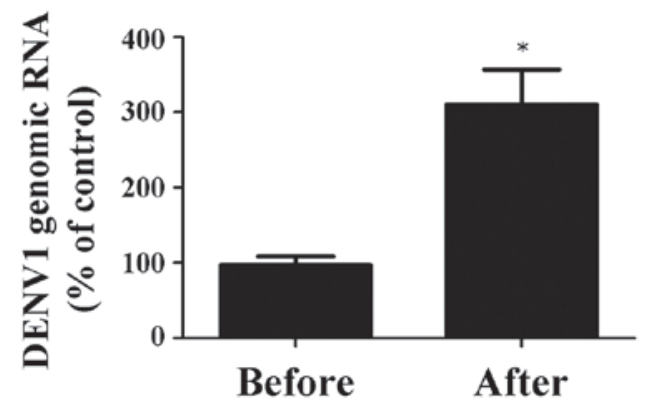

Figure 5. Concentrated genomic RNA of DENV following adsorption onto antibody-integrated magnetic beads. The culture medium $(10 \mu 1)$ of the C6/36 cells infected with DENV1 was diluted with $1 \mathrm{ml}$ phosphate-buffered saline, mixed with antibody-integrated magnetic beads and subjected to magnetic separation. Viral RNA was extracted from the samples before and after magnetic separation using a QIAamp Viral RNA Mini kit. Reverse transcription was then performed, and the resultant cDNAs were analyzed by polymerase chain reaction using forward and reverse primers for the DENV genomic RNA. The sample obtained following magnetic separation was the same as that referred to as the bead fraction. ${ }^{*} \mathrm{P}<0.05$, compared with Before. Values are presented as the mean \pm standard error. DENV, dengue virus.

of these functional units. For example, beads with a higher number of amino groups are likely to adsorb a higher number of antibody molecules, resulting in the enhanced capture and concentration of viruses. In addition, the replacement of iron, which was used in the present study, with alternative metals possessing stronger magnetic properties for the fabrication of the beads may enhance their overall capture efficiency. Furthermore, a combination of various antibodies and beads may enable the multiplex detection of pathogens.

Following the initial emergence of DENV, its spread is enhanced in a time-dependent manner. Although vaccination strategies for DENV have not been established, it is likely that infectious viruses are indispensable for future vaccine production. Therefore, the viral isolation step is essential for vaccine production. Early and efficient vaccine production is particularly important during outbreaks of DENV-associated infections. Although the early detection and isolation of DENV from mosquitoes is crucial for preventing the potential spread of disease, progress towards the development of methods for DENV detection and isolation has been limited. Ultracentrifugation and PEG precipitation are conventionally used to concentrate viruses $(8,9)$. However, these methods partially inactivate the viruses during the concentration procedure and are unsuitable for the routine monitoring of samples $(8,9)$. The possibility of using magnetic beads coated with bioadhesive molecules to concentrate viruses has been suggested previously (25-42). An example includes anionic magnetic beads coated with an anionic polymer, poly (methyl vinyl ether-maleic anhydrate), termed poly (MVE-MA), which can be used to concentrate DENV from infected mosquito cells derived from patients with dengue fever (37). Notably, the magnetic capture of other viruses using poly (MVE-MA) has been reported previously, including human immunodeficiency virus (35), borna disease virus (38), respiratory syncytial virus (39), influenza virus $(40,41)$ and adenovirus (42). The most important aspects of this method are its simplicity and rapidity $(<30 \mathrm{~min})$. Furthermore, the applicability of antibody-integrated magnetic beads to the broad serotypes 
of DENV1-4 is another promising feature of this approach. Therefore, the method for DENV capture from solution, in combination with sensitive detection methods, may contribute to preventing the spread of different subtypes of DENV. In addition, the efficient capture of infectious DENV may assist in the development of vaccines against dengue fever. The magnetic bead-concentration method may facilitate the isolation and sensitive detection of DENVs, and may contribute to efficient surveillance and future vaccine production. The general utility of the method described in the present study may be further enhanced if it is found to be applicable to other emerging viruses and bacterial pathogens.

In conclusion, the present study demonstrated that antibody-integrated magnetic beads were useful for the capture of DENVs. The capture of DENV1-4 using the antibody-integrated magnetic beads was confirmed by the results of the RT-PCR analysis, showing that the BD fraction contained DENV genomic RNA. Therefore, this method may be used in combination with conventional PCR for the detection of DENV, and may increase the sensitivity of viral detection for the diagnosis of DENV.

\section{Acknowledgements}

This study was supported by a grant for the Promotion of Basic Research Activities for Innovative Biosciences from the Bio-oriented Technology Research Advancement Institution (BRAIN), the Science and Technology Research Promotion Program for Agriculture, Forestry, Fisheries and Food Industry, the Amano Institute of Technology, and grants for Scientific Research on Innovative Areas (grant nos. 21110010 , 22110514 and 24110717) and for Scientific Research (grant no. 25246029 and 16K04997) from the Japan Society for the Promotion of Science.

\section{References}

1. World Health Organization. http://www.who.int/mediacentre/factsheets/fs117/en/ Accessed July 8, 2015.

2. Morens DM: Antibody-dependent enhancement of infection and the pathogenesis of viral disease. Clin Infect Dis 19: 500-512, 1994.

3. World Health Organization. Dengue Hemorrhagic Fever: Diagnosis, Treatment and Control. 2nd edition. World Health Organization, Geneva, 1997.

4. Sakudo A, Onodera T, Shintani H and Ikuta K: Dengue virus presence and surveillance in Okinawa (Review). Exp Ther Med 3: 15-17, 2012.

5. Hotta S: Experimental studies on dengue. I. Isolation, identification and modification of the virus. J Infect Dis 90: 1-9 1952.

6. Ministry of Health, Labour and Welfare. http://www.mhlw. go.jp/bunya/kenkou/kekkaku-kansenshou19/dengue_fever.html Accessed July 8, 2015.

7. Roth WK, Weber M and Seifried E: Feasibility and efficacy of routine PCR screening of blood donations for hepatitis $\mathrm{C}$ virus, hepatitis B virus, and HIV-1 in a blood-bank setting. Lancet 353: 359-363, 1999

8. Hamelin C and Lussier G: Concentration of human cytomegalovirus from large volumes of tissue culture fluids. J Gen Virol 42: 193-197, 1979.

9. Novotný J, Svobodová J, Ransnäs LA and Kubistová K: A method for the preparation of purified antigens of coxsackievirus B3 from a large volume of cell culture supernatant. Acta Virol 36: 483-487, 1992.

10. Safarikova $M$ and Safarik I: The application of magnetic techniques in biosciences. Magn Elect Sep 10: 223-252, 2001.
11. Pankhurst QA, Connolly J, Jones SK and Dobson J: Applications of magnetic nanoparticles in biomedicine. J Phys D Appl Phys 36: R167-R181, 2003.

12. Saraswati TE, Ogino A and Nagatsu M: Plasma-activated immobilization of biomolecules onto graphite-encapsulated magnetic nanoparticles. Carbon 50: 1253-1261, 2012.

13. Poplawska M, Bystrzejewski M, Grudziński IP, Cywińska MA, Ostapko $\mathrm{J}$ and Cieszanowski A: Immobilization of gamma globulins and polyclonal antibodies of class $\operatorname{IgG}$ onto carbon-encapsulated iron nanoparticles functionalized with various surface linkers. Carbon 74: 180-194, 2014.

14. Saraswati TE, Matsuda T, Ogino A and Nagatsu M: Surface modification of graphite encapsulated iron nanoparticles by plasma processing. Diam Relat Mater 20: 359-363, 2011.

15. Saraswati TE, Tsumura $S$ and Nagatsu M: High-efficiency plasma surface modification of graphite-encapsulated magnetic nanoparticles using a pulsed particle explosion technique. Jpn J Appl Phys 53: 010205, 2014.

16. Sakudo A, Chou H and Nagatsu M: Antibody-integrated and functionalized graphite-encapsulated magnetic beads, produced using ammonia gas plasma technology, for capturing Salmonella. Bioorg Med Chem Lett 25: 1012-1016, 2015.

17. Sakudo A, Chou H, Ikuta K and Nagatsu M: Integration of antibody by surface functionalization of graphite-encapsulated magnetic beads using ammonia gas plasma technology for capturing influenza A virus. Bioorg Med Chem Lett 25: 1876-1879, 2015.

18. Nagatsu M, Yoshida T, Mesko M, et al: Narrow multi-walled carbon nanotubes produced by chemical vapor deposition using graphene layer encapsulated catalytic metal particles. Carbon 44: 3336-3341, 2006.

19. Saito Y, Yoshikawa T, Okuda M, et al: Iron particles nesting in carbon cages grown by arc discharge. Chem Phys Lett 212 : 379-383, 1993.

20. Setthapramote C, Sasaki T, Puiprom O, Limkittikul K, Pitaksajjakul P, Pipattanaboon C, Sasayama M, Leuangwutiwong P, Phumratanaprapin W, Chamnachanan S, et al: Human monoclonal antibodies to neutralize all dengue virus serotypes using lymphocytes from patients at acute phase of the secondary infection. Biochem Biophys Res Commun 423: 867-872, 2012.

21. Masrinoul P, Diata MO, Pambudi S, Limkittikul K, Ikuta K and Kurosu T: Highly conserved region 141-168 of the NS1 protein is a new common epitope region of dengue virus. Jpn J Infect Dis 64: 109-115, 2011.

22. Paudel D, Jarman R, Limkittikul K, Klungthong C, Chamnanchanunt S, Nisalak A, Gibbons R and Chokejindachai W: Comparison of real-time SYBR green dengue assay with real-time taqman RT-PCR dengue assay and the conventional nested PCR for diagnosis of primary and secondary dengue infection. N Am J Med Sci 3: 478-485, 2011.

23. Chen $X$ and Wang J: A sequential injection fluorometric procedure for rapid determination of total protein in human serum. Talanta 69: 681-685, 2006.

24. Plapp BV, Moore S and Stein WH: Activity of bovine pancreatic deoxyribonuclease A with modified amino groups. J Biol Chem 246: 939-945, 1971

25. Safaríková M and Safarík I: Immunomagnetic separation of Escherichia coli O26, O111 and O157 from vegetables. Lett Appl Microbiol 33: 36-39, 2001.

26. Safarík I, Safaríková M and Forsythe SJ: The application of magnetic separations in applied microbiology. J Appl Bacteriol 78: 575-585, 1995.

27. Kobayashi S, Natori K, Takeda $\mathrm{N}$ and Sakae K: Immunomagnetic capture rt-PCR for detection of norovirus from foods implicated in a foodborne outbreak. Microbiol Immunol 48: 201-204, 2004.

28. Clavet CR, Margolin AB and Regan PM: Herpes simplex virus type-2 specific glycoprotein G-2 immunomagnetically captured from HEp-2 infected tissue culture extracts. J Virol Methods 119: $121-128,2004$.

29. Jothikumar N, Cliver DO and Mariam TW: Immunomagnetic capture PCR for rapid concentration and detection of hepatitis A virus from environmental samples. Appl Environ Microbiol 64: 504-508, 1998.

30. SatohK,Iwata A,MurataM,HikataM,HayakawaT and YamaguchiT: Virus concentration using polyethyleneimine-conjugated magnetic beads for improving the sensitivity of nucleic acid amplification tests. J Virol Methods 114: 11-19, 2003. 
31. Uchida E, Sato K, Iwata A, Ishii-Watabe A, Mizuguchi H, Hikata M, Murata M, Yamaguchi T and Hayakawa T: An improved method for detection of replication-competent retrovirus in retrovirus vector products. Biologicals 32: 139-146, 2004.

32. Uchida E, Kogi M, Oshizawa T, Furuta B, Satoh K, Iwata A, Murata M,Hikata M and Yamaguchi T: Optimization of the virus concentration method using polyethyleneimine-conjugated magnetic beads and its application to the detection of human hepatitis A, B and C viruses. J Virol Methods 143: 95-103, 2007.

33. Iwata A, Satoh K, Murata M, Hikata M, Hayakawa $T$ and Yamaguchi T: Virus concentration using sulfonated magnetic beads to improve sensitivity in nucleic acid amplification tests. Biol Pharm Bull 26: 1065-1069, 2003.

34. Hatano B, Kojima A, Sata T and Katano H: Virus detection using Viro-Adembeads, a rapid capture system for viruses and plaque assay in intentionally virus-contaminated beverages. Jpn J Infect Dis 63: 52-54, 2010.

35. Sakudo A and Ikuta K: A technique for capturing broad subtypes and circulating recombinant forms of HIV-1 based on anionic polymer-coated magnetic beads. Int J Mol Med 30: 437-442, 2012.
36. Sakudo A and Onodera T: Virus capture using anionic polymer-coated magnetic beads. Int J Mol Med 30: 3-7, 2012.

37. Sakudo A, Masrinoul P, Tanaka Y and Ikuta K: Capture of dengue virus type 3 using anionic polymer-coated magnetic beads. Int J Mol Med 28: 625-628, 2011.

38. Sakudo A, Tanaka Y and Ikuta K: Capture of infectious borna disease virus using anionic polymer-coated magnetic beads. Neurosci Lett 494: 237-239, 2011.

39. Sakudo A, Baba K, Tsukamoto M and Ikuta K: Use of anionic polymer, poly(methyl vinyl ether-maleic anhydride)-coated beads for capture of respiratory syncytial virus. Bioorg Med Chem Lett 19: 4488-4491, 2009.

40. Sakudo A, Baba K, Tsukamoto M, Sugimoto A, Okada T, Kobayashi T, Kawashita N, Takagi T and Ikuta K: Anionic polymer, poly(methyl vinyl ether-maleic anhydride)-coated beads-based capture of human influenza A and B virus. Bioorg Med Chem 17: 752-757, 2009.

41. Sakudo A and Ikuta K: Efficient capture of infectious H5 avian influenza virus utilizing magnetic beads coated with anionic polymer. Biochem Biophys Res Commun 377: 85-88, 2008.

42. Sakudo A, Baba K and Ikuta K: Capturing and concentrating adenovirus using magnetic anionic nanobeads. Int $\mathrm{J}$ Nanomedicine 11: 1847-1857, 2016. 